\title{
Incidencia de luxación y relación con el abordaje en una cohorte histórica de 1,738 caderas del Registro Nacional de Uruguay
}

\author{
Incidence of dislocation and relationship with the surgical approach in a \\ historic cohort of 1,738 hips of the National Register of Uruguay \\ Costa C,* Saona G, ${ }^{\ddagger}$ Rodríguez M,* Albornoz H, ${ }^{\S}$ Rey R* \\ Clínica de Traumatología y Ortopedia, Facultad de Medicina de la Universidad de la República.
}

RESUMEN. Introducción: En Uruguay se registran por ley todas las artroplastías desde 1980. La inestabilidad es la segunda causa de revisión en artroplastías de cadera. Objetivo: Conocer la incidencia de inestabilidad y su relación con el abordaje. Material y métodos: Estudio observacional y analítico de una cohorte histórica de 1,738 artroplastías de cadera efectuadas en 2014. Muestra aleatoria estratificada según la vía de abordaje: abordaje posterior (AP) y anterolateral (AAL). Se identificaron todos los pacientes que habían sufrido alguna luxación, se estimó su incidencia y se realizó análisis bivariado y multivariado para identificar los factores relacionados asociados al paciente, al cirujano (abordaje y experiencia), al medio y al implante. Resultados: 633 pacientes, seguimiento mínimo de tres años y 16 pacientes con luxación, con una incidencia de 1.95\% (IC 95\%: 1.14-3.31) en general, 1.4\% (IC 95\%: 0.643.03 ) en AAL y 4.9\% (IC 95\%: 2.67-8.83) en AP ( $p=0.009$, $\mathrm{RR}=3.35$ ). Los factores asociados con luxación fueron: a) AP con un OR de 6.18 (IC 95\%: 1.99-19.26); b) paciente procedente del subsector de salud privado con un OR de 13.74 (IC 95\%: 1.87-101.15); c) antecedente de hipotiroidismo con un OR de 3.51 (IC 95\%: 1.09-11.29); d) artrosis secundarias a artritis inflamatorias y displasia con un OR de 5.24 (IC 95\%: 1.16-23.66); e) centro quirúrgico número tres con un OR de 8.80 (IC 95\%: 1.50-51.51). Conclusiones: La incidencia de luxación temprana estuvo dentro de los rangos habituales. El AP con mayor riesgo de inestabilidad por factores de riesgo preoperatorios.

Palabras clave: Artroplastía de cadera, dislocación, inestabilidad, abordaje de cadera, artroplastía Charnley, Registro Nacional de Artroplastía.
ABSTRACT. Introduction: In Uruguay, all replacements have been registered by law since 1980 . Instability is the second cause of revision in hip replacements. Objective: To know the incidence of instability, and its relationship with the surgical approach. Material and methods: Observational and analytical study of a historical cohort of 1,738 hip replacements conducted in 2014. Random sample stratified according to the surgical approach: posterior (AP) and anterolateral (AAL). All patients who had suffered any dislocation were identified, their incidence was estimated and bivariate and multivariate tests were performed, to identify factors related, associated with the patient, to the surgeon (surgical approach and experience), to the environment, and to the implant. Results: 633 patients, minimum follow-up of three years and 16 patients with dislocation, with an incidence of 1.95\% (95\% CI: 1.14-3.31) in general, $1.4 \%$ (95\% CI: 0.64-3.03) in AAL and 4.9\% (95\% CI: 2.67-8.83) in AP (p = 0.009 , RR $=3.35$ ). Factors associated with dislocation were: a) AP with an OR of 6.18 (CI 95\%: 1.99-19.26); b) patient from the private health subsector with an OR of 13.74 (95\% CI: 1.87-101.15); c) antecedent of hypothyroidism with an OR of 3.51 (IC 95\%: 1.09-11.29); d) osteoarthritis secondary to inflammatory arthritis and dysplasia with an OR of 5.24 (CI 95\%: 1.16-23.66); e) surgical center number three with an OR of 8.80 (CI 95\%: 1.50-51.51). Conclusions: The incidence of early dislocation was within the usual ranges. Posterior surgical approach with increased risk of instability from preoperative risk factors.

Keywords: Hip arthroplasty, dislocation, instability, hip approach, Charnley arthroplasty, Arthroplasty National Register.

\section{Nivel de evidencia: II}

* Clínica de Traumatología y Ortopedia, Facultad de Medicina de la Universidad de la República. Montevideo, Uruguay.

${ }^{\ddagger}$ Fondo Nacional de Recursos. Montevideo, Uruguay.

$\S$ Cátedra de Enfermedades Infecciosas de la Universidad de la República. Montevideo, Uruguay.

Correspondencia:

Dr. Rogelio Rey

Chiávari 3009, C.P. 11600, Montevideo, Uruguay.

E-mail: drrogeliorey@gmail.com / traumatologia@fmed.edu.uy

Recibido: 10-08-2020. Aceptado: 10-02-2021.

Citar como: Costa C, Saona G, Rodríguez M, Albornoz H, Rey R. Incidencia de luxación y relación con el abordaje en una cohorte histórica de 1,738 caderas del Registro Nacional de Uruguay. Acta Ortop Mex. 2021; 35(1): 51-55. https://dx.doi.org/10.35366/100931 
Introducción

Sir John Charnley introdujo hace más de 50 años la prótesis total de cadera de baja fricción, lo que revolucionó el tratamiento de la artrosis de cadera, convirtiéndose en el patrón de oro del tratamiento de esta patología y por lo general con excelentes resultados, ${ }^{1}$ con un riesgo de revisión que es por lo regular menor de $5 \%$ a 10 años, ${ }^{2}$ pero los datos existentes no son suficientes para conocer exactamente cuánto va a durar una cadera protésica, incluso con implantes de última generación. ${ }^{3}$

Es un procedimiento no exento de complicaciones y de ellas, la luxación es la segunda complicación más frecuente, luego del aflojamiento aséptico, con una incidencia variable, generando una morbilidad e impacto socioeconómico importante; $;, 5$ y con muchos factores que se mencionan como propiciadores de la misma, incluyendo los que dependen del implante, del cirujano, del paciente y de la técnica quirúrgica. ${ }^{4,5,6}$

Dentro de la asociación de factores no parecen existir dudas de que el abordaje posterolateral y las cabezas pequeñas (fundamentalmente 22) 4,5,6 $^{4}$ tienen mayor riesgo de luxación temprana, una combinación utilizada con frecuencia en nuestro país. En tal sentido, usar cabezas de gran diámetro, más de $38 \mathrm{~mm}$, no afectan la incidencia de riesgo.

En 1980 se dictó en Uruguay la Ley 14897 y se creó así un fondo público no estatal denominado Fondo Nacional de Recursos (FNR), que permite mediante una pequeña cuota ( $<7$ US\$/mes), aportada por los habitantes contribuyentes del Fondo Nacional de Salud y por el Ministerio de Economía y Finanzas, dar cobertura total a todos los procedimientos de alto costo (transplantes, medicación oncológica y biológica, cirugía cardíaca, hemodiálisis, artroplastías de cadera y rodilla, entre otros). Esto permite centralizar y registrar todas las cerca de 4,000 artroplastías de cadera anuales realizadas en cinco centros quirúrgicos, denominados Institutos de Medicina Altamente Especializados (IMAE), todos con salas de flujo laminar, independientemente de que el paciente provenga del sector público o privado y no se realizan en ninguna otra sala de operaciones. El registro es obligatorio para el pago del procedimiento. ${ }^{7,8}$ El sistema paga un implante del tipo prótesis de Charnley cementadas monoblock de origen argentino, con cabeza 22.225, que en 2014 eran más de 80\% de los casos. El paciente puede acceder a otros implantes (diferente cabeza, sistema de fijación y superficie de fricción), siempre y cuando pague el costo del mismo.

El objetivo de este trabajo fue analizar, a través del Registro Nacional de Artroplastías del Fondo Nacional de Recursos (FNR), la incidencia real de inestabilidad (luxación) en una cohorte histórica de los pacientes operados de todo el país (Uruguay) por artrosis en el año 2014 y evaluar la presencia de factores que la propician, haciendo especial énfasis en la vía de abordaje y su asociación con cabezas pequeñas.

\section{Material y métodos}

En el año 2014 se operaron en Uruguay un total de 1,738 artroplastías de caderas primarias por artrosis por unos 150 cirujanos con diferente experiencia. En dicha cohorte de pacientes se decidió realizar un estudio analítico, observacional, recabando información de forma prospectiva (base de datos clínicos del FNR) y retrospectivo (entrevista telefónica). Teniendo en cuenta el objetivo, se seleccionaron dos poblaciones dependiendo del abordaje utilizado, posterior (AP: 274 caderas) o anterolateral (AAL: 1,464 caderas).

Con la población categorizada, se tomó una muestra representativa para ambos abordajes, teniendo como hipótesis una incidencia de luxación de $2 \%$ en AAL y $6 \%$ en AP. Se obtuvo una muestra final de 681 pacientes de los que se descartaron 48, porque algunos datos de la Historia Clínica Electrónica (base de datos del FNR) no eran fidedignos y la mayoría estaban muertos y no se podían evaluar. La muestra final evaluable fue de 633 pacientes y se consideró representativa al ser $93 \%$ de la muestra seleccionada y con características homogéneas con la población total para el año en estudio (Tabla 1).

En la muestra de 633 pacientes, 204 fueron por AP y 429 por AAL, la ponderación correspondiente para cada grupo fue de $1.34(204 / 274)$ para el AP y de $3.41(429 / 1,464)$ para la AAL.

Mediante el registro digital de los datos clínicos de los pacientes aportados por la base de datos del FNR y el llamado telefónico por parte de dos residentes de traumatología a todos los pacientes seleccionados, entre Septiembre de 2017 y Enero de 2018 se construyó la tabla de recolección de datos previamente diseñada, destinada a evaluar episodios de luxación, mecanismo de la misma, número y tratamientos realizados.

Se evaluaron factores demográficos y clínicos de los pacientes así como la vía de abordaje, el tamaño de la cabeza utilizado y la experiencia del cirujano, considerando ésta si operaba menos de 10, entre 10 y 49 y más de 50 prótesis por año, respectivamente.

El análisis estadístico consistió en la descripción de las variables cuantitativas por medio de media y desvío estándar y las cualitativas utilizando porcentajes u odds ratio (OR). En la estimación de proporciones se calculó el intervalo de confianza de $95 \%$ utilizando el método de Wilson. En las comparaciones de proporciones se utilizó el test de $\chi^{2}$ y en la comparación de medias el test de Student.

Se utilizó la regresión logística binaria para evaluar la asociación entre el conjunto de factores preoperatorios y de la cirugía (institución, experiencia del cirujano, abordaje, tamaño de prótesis) con la incidencia de luxación. En el modelo inicial se consideraron todas las variables y fueron retiradas en orden descendente del valor p. Además, se tomó en cuenta el efecto de confusión de una variable, si su presencia contribuía a hacer significativo el efecto de otra. Para corregir problemas numéricos en variables con frecuencia 


\begin{tabular}{|c|c|c|}
\hline \multicolumn{3}{|c|}{$\begin{array}{l}\text { Tabla 1: Comparación entre las características } \\
\text { en la población total y la población reconstruida } \\
\text { mediante la ponderación de la muestra. }\end{array}$} \\
\hline & $\begin{array}{c}\text { Población total } \\
\text { n (\%) }\end{array}$ & $\begin{array}{c}\text { Población reconstruida } \\
\text { n (\%) }\end{array}$ \\
\hline Edad (años)* & $68.4 \pm 11.2$ & $68.3 \pm 10.9$ \\
\hline Sexo masculino & 687 (39.5) & 693 (39.8) \\
\hline $\mathrm{AP}$ & 274 (15.8) & $274(15.8)$ \\
\hline AAL & $1,465(84.2)$ & $1,465(84.2)$ \\
\hline \multicolumn{3}{|l|}{ IMAE } \\
\hline 1 & 185 (10.6) & $192(11.0)$ \\
\hline 2 & 777 (44.7) & 815 (46.9) \\
\hline 3 & $250(14.4)$ & 235 (13.5) \\
\hline 4 & $35(2.0)$ & $41(2.4)$ \\
\hline 5 & 492 (28.3) & $456(26.2)$ \\
\hline \multicolumn{3}{|c|}{$\begin{array}{l}\text { AP = abordaje posterior; } \mathrm{AAL}=\text { anterolateral; IMAE = Institutos de Medicina } \\
\text { Altamente Especializados. } \\
\text { * Media } \pm \text { desviación estándar. }\end{array}$} \\
\hline
\end{tabular}

cero o muy baja se agruparon categorías (p. ej.: IMAE y artrosis).

En todas las estimaciones se tuvo en cuenta el diseño de muestreo estratificado al azar y se utilizaron los pesos de los estratos.

Se consideraron significativos los valores $\mathrm{p}$ mayores o iguales a 0.05 y en el análisis se utilizó el programa estadístico Stata 16.1.

\section{Resultados}

La muestra aleatoria final de 633 pacientes presentó características muy similares de la población de 1,738 artroplastías realizadas durante 2014 considerándose por tanto representativa (Tabla 1). La mediana de seguimiento fue de 3.35 años (de 33 a 45 meses). En dicho período 16 pacientes mostraron algún episodio de luxación, de los cuales seis tenían AAL y 10 AP, representando una incidencia global para la población general de 1.95\% (IC 95\% 1.14-3.31); siendo de 1.4\% (IC 95\% 0.64-3.03) para AAL y de 4.9\% (IC 95\% 2.67-8.83) para AP, presentando una diferencia estadísticamente significativa para ambos abordajes $(\mathrm{p}=0.009, \mathrm{RR}=3.35)$.

En la Tabla 2 se describe la significación estadística en la asociación con el evento de luxación del análisis bivariado, destacándose las variables significativas: subsector de salud e IMAE. En este análisis la experiencia del cirujano mostró diferencias en el total de la muestra y también en el AAL, siendo menor para los cirujanos de mayor experiencia, pero con valores sin significancia estadística.

En la Tabla 3 se muestra el resultado del análisis multivariado mediante regresión logística binaria, se presentan dos modelos que se diferencian por la incorporación de la variable Centro Quirúrgico o IMAE (modelo II) o no (modelo I). La incorporación de esta variable IMAE al modelo I hace que la vía de abordaje pierda significación estadística $(p=0.137)$ y que el hipotiroidismo resulte significativo $(p$ $=0.035$ ).
Existen algunos factores de riesgo, conocidos como propiciadores de luxación, pero que en esta serie no fueron estadísticamente significativos como la edad, el sexo, el índice de masa corporal (IMC) y los antecedentes neurológicos (Tablas 2 y 3). Sin embargo, fue necesario incluir la edad, el sexo y su interacción en los modelos logísticos para ajustar el efecto de confusión y que resultaran significativos el hipotiroidismo y la artrosis secundaria a displasia y artritis.

\begin{tabular}{|c|c|c|c|}
\hline \multicolumn{4}{|c|}{$\begin{array}{l}\text { Tabla 2: Análisis bivariado de factores asociados } \\
\text { con la luxación del paciente. Para simplificar la } \\
\text { tabla las celdas vacías corresponden a proporciones } \\
\text { complementarias de las de luxación. }\end{array}$} \\
\hline & $\begin{array}{c}\text { Luxación } \\
\%\end{array}$ & $\begin{array}{c}\text { No luxación } \\
\%\end{array}$ & $\mathrm{p}$ \\
\hline Edad (años) & $67.5 \pm 14.5$ & $68.0 \pm 11.6$ & 0.892 \\
\hline IMC $\left(\mathrm{kg} / \mathrm{m}^{2}\right)$ & $27.3 \pm 4.3$ & $28.3 \pm 4.6$ & 0.385 \\
\hline \multicolumn{4}{|l|}{ Sexo } \\
\hline Femenino & 2.06 & & 0.079 \\
\hline Masculino & 1.76 & & \\
\hline \multicolumn{4}{|l|}{ Subsector salud } \\
\hline Público & 0.34 & & 0.027 \\
\hline Privado & 2.42 & & \\
\hline \multicolumn{4}{|l|}{ Región } \\
\hline Montevideo & 2.66 & & 0.242 \\
\hline Interior & 1.36 & & \\
\hline Hipotiroidismo & 3.94 & 1.63 & 0.146 \\
\hline Neurológicos & 0.00 & 2.10 & 0.122 \\
\hline \multicolumn{4}{|l|}{ ASA } \\
\hline 1 & 0.00 & & 0.897 \\
\hline 2 & 2.05 & & \\
\hline 3 & 0.00 & & \\
\hline 4 & 0.00 & & \\
\hline \multicolumn{4}{|l|}{ Artrosis } \\
\hline Primaria & 1.76 & & 0.282 \\
\hline $\begin{array}{l}\text { Secundaria } \\
\text { displasia }\end{array}$ & 6.26 & & \\
\hline $\begin{array}{l}\text { Secundaria } \\
\text { artritis }\end{array}$ & 6.80 & & \\
\hline $\begin{array}{l}\text { Secundaria } \\
\text { necrosis }\end{array}$ & 1.72 & & \\
\hline \multicolumn{4}{|l|}{ Tamaño de cabeza } \\
\hline $22 \mathrm{~mm}$ & 2.07 & & 0.525 \\
\hline 28 mm o más & 1.37 & & \\
\hline \multicolumn{4}{|l|}{ Abordaje } \\
\hline AP & 4.90 & & 0.009 \\
\hline AAL & 1.40 & & \\
\hline \multicolumn{4}{|l|}{$\begin{array}{l}\text { Experiencia del } \\
\text { cirujano }\end{array}$} \\
\hline $\begin{array}{l}\text { Menos de } 10 \\
\text { cirugías/año }\end{array}$ & 3.25 & & 0.824 \\
\hline $\begin{array}{l}\text { De } 11 \text { a } 50 \\
\text { cirugías/año }\end{array}$ & 2.07 & & \\
\hline $\begin{array}{l}\text { Más de } 50 \\
\text { cirugías/año }\end{array}$ & 1.76 & & \\
\hline \multicolumn{4}{|l|}{ IMAE } \\
\hline 1 & 1.42 & & 0.018 \\
\hline 2 & 4.96 & & \\
\hline 3 & 8.33 & & \\
\hline 4 & 4.00 & & \\
\hline 5 & 0.00 & & \\
\hline $\begin{array}{l}\text { IMC = índice de mas } \\
\text { AP = abordaje poste } \\
\text { Altamente Especializ }\end{array}$ & $\begin{array}{l}\text { poral; } \mathrm{ASA}=\mathrm{A} \\
\mathrm{AL}=\text { anterolat }\end{array}$ & $\begin{array}{l}\text { ican Society of } \\
\text {; IMAE = Instit }\end{array}$ & $\begin{array}{l}\text { siologists; } \\
\text { Medicina }\end{array}$ \\
\hline
\end{tabular}




\begin{tabular}{|c|c|c|c|c|}
\hline & \multicolumn{2}{|c|}{ Modelo I } & \multicolumn{2}{|c|}{ Modelo II } \\
\hline & OR (IC 95\%) & $\mathrm{p}$ & OR (IC 95\%) & $\mathrm{p}$ \\
\hline Edad (años) & $0.98(0.93-1.04)$ & 0.568 & $1.00(0.95-1.05)$ & 0.899 \\
\hline Sexo masculino ${ }^{\mathrm{a}}$ & $0.06(0.00-92.00)$ & 0.457 & $0.18(0.00-135.91)$ & 0.615 \\
\hline Edad \# Sexo M & $1.04(0.94-1.15)$ & 0.452 & $1.02(0.93-1.13)$ & 0.620 \\
\hline Subsector Salud Privadab & $13.74(1.87-101.15)$ & 0.010 & $11.32(1.54-83.29)$ & 0.017 \\
\hline Hipotiroidismo & $3.01(0.92-9.83)$ & 0.068 & $3.51(1.09-11.29)$ & 0.035 \\
\hline Artrosis secundaria displasia 0 artritis ${ }^{c}$ & $5.24(1.16-23.66)$ & 0.031 & $5.95(1.40-25.37)$ & 0.016 \\
\hline Abordaje AP ${ }^{d}$ & $6.18(1.99-19.26)$ & 0.002 & $3.52(0.67-18.56)$ & 0.137 \\
\hline \multicolumn{5}{|l|}{ IMAE } \\
\hline $2^{\mathrm{e}}$ & Variable no incluida & & $4.00(0.66-24.40)$ & 0.133 \\
\hline 3 & & & $8.80(1.50-51.51)$ & 0.016 \\
\hline 4 & & & $2.56(0.45-14.62)$ & 0.289 \\
\hline Constante & $<0.01$ & 0.001 & $<0.01$ & $<0.01$ \\
\hline
\end{tabular}

De los 16 pacientes que sufrieron luxación, nueve tuvieron un único episodio de luxación, tres pacientes dos episodios y cuatro pacientes tres o más episodios. El mecanismo de luxación fue la hiperflexión del tronco sobre el muslo en 11 de ellos (10 al agacharse y uno en asiento bajo) y en cinco no fue claro (cuatro acostados y uno traumático). Podemos deducir que la mayoría fueron luxaciones posteriores y sólo de los que estaban acostados podemos dudar del mecanismo y del sentido de la luxación. Todos los episodios de luxación requirieron reducción bajo anestesia general en block quirúrgico y no hubo ninguna revisión hasta el momento. Cinco de los pacientes recibieron tratamiento ortopédico (cuatro con rodillera de yeso por seis semanas y un caso, pelvicazón de yeso).

\section{Discusión}

La incidencia de luxación protésica publicada mundialmente es muy variable, entre 0.5 y $9.2 \% .{ }^{4}$ Por ejemplo, el Registro Nacional de Escocia sobre 14,000 artroplastías publica $1.9 \%$ al año y así como aumenta en mayores de 85 años, disminuye si el cirujano tiene entre 11 y 25 procedimientos por año. ${ }^{10}$

Kornuijt dice que el abordaje posterior es seguro si se toman medidas correctas de cierre y las cabezas tienen entre 28 y $36 \mathrm{~mm} \cdot{ }^{11}$ A pesar de esto, Sheth ya señaló hace unos años en un registro de más de 42,000 reemplazos de cadera, que los abordajes anterior y anterolateral tenían muy bajo índice de revisión por luxación. ${ }^{12}$

Sigue teniendo total vigencia la evaluación de la colocación de los componentes y la safe zone (zona de seguridad), según Lewinnek, desde $1978^{13}$ e incluso, Callanan relaciona el posicionamiento acetabular con algunos de los elementos que se mencionan como propiciadores de luxación, fundamentalmente las miniincisiones, la escasa experiencia del cirujano y los pacientes obesos. ${ }^{14}$
En esta serie, que incluye una muestra representativa de todos los pacientes operados durante un año por cirujanos del país y en su mayoría con tallos monoblock con cabeza 22.225 , las cifras globales de $1.9 \%$ son similares a las publicadas en la literatura. Y si evaluamos la relación con los abordajes, las incidencias de 1.4\% para AAL y de $4.9 \%$ para AP también son similares a las publicadas, pero con una diferencia significativa entre las mismas para el tipo de implante que se utiliza en nuestro país (fundamentalmente cabezas pequeñas).

Existen dos elementos que presentaron significancia en este trabajo, cuya explicación podría no ser clara y que no tiene antecedentes similares de publicación. Los pacientes del subsector público se luxan menos porque pueden elegir cirujanos más experimentados; tienen consultas de prevención preoperatorias y la demora en sus estudios los hace llegar con mas información preoperatoria. La diferencia significativa, según el centro quirúrgico, está asociada a cirujanos menos experimentados o a una preferencia por el abordaje posterior.

Queremos señalar, por ser un dato original, la diferencia estadísticamente significativa con relación al hipotiroidismo y su aumento de riesgo de luxación, ya que la relación de las muestras con respecto a esta patología previa es similar. No encontramos esta asociación previamente en la literatura, aunque sí es una patología vinculada a mayor riesgo de infecciones. ${ }^{15}$

A diferencia de otros estudios, en nuestro trabajo no hubo diferencias estadísticamente significativas en variables como edad, sexo, índice de masa corporal, patologías neurológicas y ASA. ${ }^{9,16,17}$ La falta de significación en dichas variables puede deberse al bajo número de eventos de luxación en relación al número de variables consideradas en el análisis multivariado o quizás influya positivamente la formación de los equipos quirúrgicos en estos resultados y las medidas de prevención que se toman en la enseñanza de conductas para con los pacientes. 


\section{Conclusiones}

Nuestra incidencia se encuentra en los rangos habituales publicados en la bibliografía, el registro incluye a todos los cirujanos del país y la mayoría de los implantes utilizados son prótesis de Charnley monoblock con cabeza $22.225 \mathrm{~mm}$.

El abordaje posterolateral presenta una incidencia de luxaciones más de tres veces superior al abordaje anterolateral y confirma que la asociación de este abordaje y cabeza pequeña no debe utilizarse, incluso en cirujanos expertos.

Referencias

1. Learmonth ID, Young C, Rorabeck C. The operation of the century: total hip replacement. Lancet. 2007; 370(9597): 1508-19.

2. NICE. Total hip replacement and resurfacing arthroplasty for end stage arthritis of the hip. NICE technology appraisal guidance 304. London: National Institute for Health and Care Excellence; 2014.

3. Evans JT, Evans JP, Walker RW, Blom AW, Whitehouse MR, Sayers A. How long does a hip replacement last? A systematic review and meta-analysis of case series and national registry reports with more than 15 years of follow-up. Lancet. 2019; 393(10172): 647-54.

4. Dabaghi A, Saleme J, Ochoa L. Evaluación y tratamiento de la luxación protésica de cadera. Acta Ortop Mex. 2014; 28(2): 137-44.

5. Fernández-Fairen M, Hernández-Vaquero D, Murcia-Mazón A, Querales-Leal V, Torres-Pérez AI, Murcia-Asensio A. Inestabilidad de la artroplastía total de cadera. Una aproximación desde los criterios de la evidencia científica. Rev Esp Cir Ortop Traumatol. 2011; 55(6): 460-75.

6. Kumar V, Sharma S, James J, Hodgkinson JP, Hemmady MV. Total hip replacement through a posterior approach using a $22 \mathrm{~mm}$ diameter femoral head: the role of the transverse acetabular ligament and capsular repair in reducing the rate of dislocation. Bone Joint J. 2014; 96-B(9): 1202-6.

7. Fondo Nacional de Recursos Medicina Altamente Especializada. [consultado 1 Ago 2017] Disponible en: http://www.fnr.gub.uy/ tecnicas beneficiarios
8. Ibarra Melogno S, Chifflet L, Rey R, Leiva G, Morales N, Albornoz $H$. Resultados a largo plazo de artroplastía de cadera en pacientes en diálisis por insuficiencia renal crónica. Mortalidad y supervivencia del implante en el Registro Nacional de Uruguay desde el año 2000. Rev Esp Cir Ortop Traumatol. 2019; 63(3): 187-91. https://doi. org/10.1016/j.recot.2018.12.002

9. Thompson SK. Sampling. 2nd edition. United States: John Wiley \& Sons; 2002. p. 367.

10. Meek RM, Allan DB, McPhillips G, Kerr L, Howie CR. Epidemiology of dislocation after total hip arthroplasty. Clin Orthop Relat Res. 2006; 447: 9-18.

11. Kornuijt A, Das D, Sijbesma T, van der Weegen W. The rate of dislocation is not increased when minimal precautions are used after total hip arthroplasty using the posterolateral approach: a prospective, comparative safety study. Bone Joint J. 2016; 98-B(5): 589-94.

12. Sheth D, Cafri G, Inacio MC, Paxton EW, Namba RS. Anterior and anterolateral approaches for THA are associated with lower dislocation risk without higher revision risk. Clin Orthop Relat Res. 2015; 473(11): 3401-8.

13. Lewinnek GE, Lewis JL, Tarr R, Compere CL, Zimmerman JR. Dislocations after total hip-replacement arthroplasties. J Bone Joint Surg Am. 1978; 60 (2): 217-220.

14. Callanan MC, Jarrett B, Bragdon CR, Zurakowski D, Rubash HE, Freiberg AA, et al. The John Charnley Award: risk factors for cup malpositioning: quality improvement through a joint registry at a tertiary hospital. Clin Orthop Relat Res. 2011; 469(2): 319-29.

15. Tan TL, Rajeswaran H, Haddad S, Shahi A, Parvizi J. Increased risk of periprosthetic joint infections in patients with hypothyroidism undergoing total joint arthroplasty. J Arthroplasty. 2016; 31(4): 86871.

16. Tansey RJ, Green GL, Haddad FS. Large diameter heads: is bigger always better? Semin Arthroplasty. 2015; 26(1): 16-9.

17. Jolles BM, Zangger P, Leyvraz PF. Factors predisposing to dislocation after primary total hip arthroplasty: a multivariate analysis. $J$ Arthroplasty. 2002; 17(3): 282-8.

Fuentes de financiación: Clínica de Traumatología y Ortopedia y el Fondo Nacional de Recursos, Montevideo, Uruguay.

Conflicto de intereses: Los autores declaran no tener conflicto de intereses. 